DAMTP-R94/30

gr-qc/9408013

10th August, 1994

\title{
Spin networks, Turaev-Viro theory and the loop representation.
}

\author{
Timothy J. Foxon ${ }^{\dagger}$ \\ Department of Applied Mathematics and Theoretical Physics, \\ University of Cambridge, \\ Silver Street, Cambridge, CB3 9EW, U.K.
}

\begin{abstract}
.
We investigate the Ponzano-Regge and Turaev-Viro topological field theories using spin networks and their $q$-deformed analogues. I propose a new description of the state space for the Turaev-Viro theory in terms of skein space, to which $q$-spin networks belong, and give a similar description of the Ponzano-Regge state space using spin networks. I give a definition of the inner product on the skein space and show that this corresponds to the topological inner product, defined as the manifold invariant for the union of two 3-manifolds.

Finally, we look at the relation with the loop representation of quantum general relativity, due to Rovelli and Smolin, and suggest that the above inner product may define an inner product on the loop state space.
\end{abstract}

$\dagger$ e-mail: tjf12@amtp.cam.ac.uk 


\section{Introduction.}

In this paper, we use the ideas of spin networks to examine the relation between certain discrete models for gravity, based on topological field theories, and the loop representation of quantum general relativity, based on Ashtekar variables.

Spin networks were introduced by Penrose [1971] in an attempt to use combinatorial ideas to construct a discrete model for a quantum mechanical structure underlying classical space-time. This model anticipates many of the ideas that were later formalised in topological field theories. A spin network is an $S U(2)$ invariant tensor represented by a trivalent graph. In such a network, three labelled edges meet at each vertex, where each edge is labelled by a half-integer representation $j_{i}$ of the group $S U(2)$.

A 3-dimensional topological field theory is defined [Atiyah 1990] by the assignment of a topological invariant to a closed 3-manifold and a vector space to a 2-dimensional surface. These assignments are specified by defining the partition function of the theory. We consider, in particular, topological field theories originally defined on a triangulated 3manifold by a partition function which is a sum over states on the interior of the manifold. A state is defined by labelling or 'colouring' each edge of the triangulation by a half-integer representation $j_{i}$ of the classical group $S U(2)$ [Ponzano and Regge 1968] or the quantum group $U_{q}(\operatorname{sl}(2))$ at $q$ an $r$-th root of unity [Turaev and Viro 1992].

We begin, in section 2, by defining the state space and the inner product for the Regge-Ponzano model and reviewing the previous work of Ooguri [1992]. In section 3, I show that there is a natural interpretation in terms of a spin network picture and extend the work of Rovelli [1993] to relate this to the loop representation [Rovelli and Smolin 1990]. In section 4, I describe a conjectured new description of the state space of the Turaev-Viro theory, obtained using the $q$-deformed version of spin networks [Kauffman 1991], and re-write the inner product in this picture. Finally, in section 5, we discuss the

possible implications of these ideas, and make the tenative proposal that the Turaev-Viro theory may be identified as a naturally regularised version of the loop representation. 


\section{State space for Regge-Ponzano theory.}

The topological field theory of Turaev and Viro [1992] is defined by taking a triangulation of a 3-manifold $M$, on which a state or colouring is given by labelling each edge of the triangulation by a representation $j_{i}$ of the quantum group $U_{q}(\operatorname{sl}(2))$. The partition function $Z_{T V}(M)$ for the manifold $M$ is given by a finite sum over states on the interior of the manifold of the product of $q-6 j$ symbols $\left\{6 j^{(t)}\right\}_{q}$ corresponding to tetrahedra $t$, weighted by specified functions $w_{v}\left(j_{i}\right)$ for each vertex, $w_{e}\left(j_{i}\right)$ for each internal edge, and $f\left(c_{j}\right)$ for the colouring $c_{j}$ on the boundary,

$$
Z_{T V}\left(M, c_{j}\right)=\sum_{\text {states },\left\{j_{i}\right\}} f\left(c_{j}\right) \prod_{\text {vertices }, v} w_{v}\left(j_{i}\right) \prod_{\text {edges }, e} w_{e}\left(j_{i}\right) \prod_{\text {tetra, },}\left\{\begin{array}{lll}
j_{1}^{(t)} & j_{2}^{(t)} & j_{3}^{(t)} \\
j_{4}^{(t)} & j_{5}^{(t)} & j_{6}^{(t)}
\end{array}\right\}_{q},
$$

for states which satisfy certain admissibility conditions, as we shall discuss below. For a closed 3-manifold, this partition function is a topological invariant, i.e. it depends only on the topology of the manifold $M$ and not on the triangulation used in the definition. For a 3-manifold with boundary, the partition function is a function of the colouring on the boundary, but still depends only on the topology of the interior of the manifold.

In the $q \rightarrow 1$ limit, this reduces to the much earlier theory of Ponzano and Regge [1968], in which the $j_{i}$ label representations of the classical group $S U(2)$. The ReggePonzano partition function $Z_{R P}(M)$ has a similar form to $(2.1)$ but with the $\left\{6 j^{(t)}\right\}_{q}$ replaced by classical $6 j$ symbols $\left\{6 j^{(t)}\right\}$. Specificly, for manifold $M$ with colouring $c_{j}$ on its boundary $F$,

$$
\begin{aligned}
Z_{R P}\left(M, c_{j}\right)= & \sum_{\text {states, }\left\{j_{i}\right\}} \prod_{\text {edges } \in F, k}(-1)^{2 j_{k}} \sqrt{2 j_{k}+1} \prod_{\text {vertices }, v} \Lambda(j)^{-1} \\
\prod_{\text {edges }, i}\left(2 j_{i}+1\right) & \prod_{\text {tetra }, t}(-1)^{\sum j_{i}}\left\{\begin{array}{lll}
j_{1}^{(t)} & j_{2}^{(t)} & j_{3}^{(t)} \\
j_{4}^{(t)} & j_{5}^{(t)} & j_{6}^{(t)}
\end{array}\right\} .
\end{aligned}
$$

In this case, the state sum is infinite, but it may be regularised by truncating the sum at a large value $L$ of $j_{i}$, and the taking the limit as $L \rightarrow \infty$. In this section, we shall assume that this may be done, and interpret the Regge-Ponzano theory as a formal topological field theory.

To define fully a 3-dimensional topological field theory [Atiyah 1990], the vector space of states $V(F)$ assigned to a 2-dimensional surface $F$ must also be specified. We define the vector space $V_{R P}(F)$ for Regge-Ponzano theory by analogy with the Turaev-Viro definition. This is essentially equivalent to that given by Ooguri [1992], but written in a way that allows us to give a simple definition of the inner product. The vector space $V_{R P}(F)$ 
associated to a surface $F$, triangulated by $\Delta$, is defined as a quotient of the space $C(F, \Delta)$ of linear combinations of colourings of the triangulated surface, given by

$$
\begin{aligned}
V_{R P}(F) & =\left\{\phi_{\Delta}=\sum_{i} \lambda_{i}\left|c_{i}\right\rangle: \mathrm{P}\left[\phi_{\Delta}\right]=\phi_{\Delta}\right\} \\
& =C(F, \Delta) /\left\{\phi_{\Delta}: \mathrm{P}\left[\phi_{\Delta}\right]=0\right\},
\end{aligned}
$$

where $\mathrm{P}$ is the projection operator corresponding to the cylinder $F \times[0,1]$ over the surface, given by

$$
\mathrm{P}\left|c_{i}\right\rangle=\sum_{c_{i}^{\prime}} Z\left(F \times I, c_{i}, c_{i}^{\prime}\right)\left|c_{i}^{\prime}\right\rangle .
$$

It is natural to assign the vector $|M\rangle \in V_{R P}(F)$ associated to a 3-manifold $M$ with boundary $\partial M=F$ as

$$
|M\rangle=\sum_{c_{i}} Z_{R P}\left(M, c_{i}\right)\left|c_{i}\right\rangle
$$

where $c_{i}$ is a colouring of the boundary, and $Z_{R P}\left(M, c_{i}\right)$ is the partition function given by a state sum of the form (2.2) over all colourings of the interior which extend the colouring $c_{i}$ on the boundary.

An inner product may be defined on the space of colourings $C(F, \Delta)$ by taking the set of different colourings on $F$ to form an orthonormal basis,

$$
\left\langle c_{i} \mid c_{j}\right\rangle=\delta_{i j}
$$

On the quotient space $V_{R P}(F)$, we define the inner product to be

$$
\left(\xi_{i}, \xi_{j}\right)=\left\langle\xi_{i} \mid \mathrm{P}\left[\xi_{j}\right]\right\rangle
$$

so that, for $\phi, \eta \in\left\{\phi_{\Delta}: \mathrm{P}\left[\phi_{\Delta}\right]=0\right\}$, as $\mathrm{P}$ is self-adjoint under (2.6), we have

$$
\begin{aligned}
\left(\xi_{i}+\phi, \xi_{j}+\eta\right) & =\left\langle\xi_{i}+\phi \mid \mathrm{P}\left[\xi_{j}+\eta\right]\right\rangle \\
& =\left\langle\xi_{i} \mid \mathrm{P}\left[\xi_{j}\right]\right\rangle+\left\langle\phi \mid \mathrm{P}\left[\xi_{j}\right]\right\rangle \\
& =\left\langle\xi_{i} \mid \mathrm{P}\left[\xi_{j}\right]\right\rangle+\overline{\left\langle\xi_{j} \mid \mathrm{P}[\phi]\right\rangle} \\
& =\left(\xi_{i}, \xi_{j}\right)
\end{aligned}
$$

and so the inner product is well-defined on $V_{R P}(F)$.

The above definitions imply that the inner product between the two vectors corresponding to two 3 -manifolds $M_{1}, M_{2}$, which meet in a common surface $F$, is given by the 
invariant of the manifold obtained by gluing $M_{1}$ and $M_{2}$ along $F$, as follows

$$
\begin{aligned}
\left(M_{2}, M_{1}\right) & =\sum_{c_{i}, c_{j}, c_{i}^{\prime}} \overline{Z\left(M_{2}, c_{i}\right)} Z\left(F \times I, c_{j}, c_{i}^{\prime}\right) Z\left(M_{1}, c_{j}\right)\left\langle c_{i} \mid c_{i}^{\prime}\right\rangle \\
& =\sum_{c_{i}, c_{j}} \overline{Z\left(M_{2}, c_{i}\right)} Z\left(F \times I, c_{j}, c_{i}\right) Z\left(M_{1}, c_{j}\right) \\
& =Z_{R P}\left(M_{2} \cup M_{1}\right) .
\end{aligned}
$$

This is the definition of the inner product required for a topological field theory [Atiyah 1990] to be consistent, and so this definition carries a lot of information about the elements of the theory.

Witten [1989a] defined a set of topological field theories in which the partition function $Z_{C S W}(M)$ is given by a Chern-Simons path integral

$$
Z_{C S W}(M)=\int[\mathrm{d} A] \exp \left(i \frac{k}{4 \pi} \int_{M} \operatorname{Tr}\left(A \wedge \mathrm{d} A+\frac{2}{3} A \wedge A \wedge A\right)\right)
$$

with the connection $A$ taking values in the Lie algebras of various gauge groups. He argued [Witten 1989b,c] that Chern-Simons theories with certain choices of gauge group are equivalent to 2+1-dimensional gravity with or without cosmological constant. It follows that the Chern-Simons theory with gauge group $I S O(3)$ is equivalent to 3 -dimensional Euclidean gravity without cosmological constant. This theory is related to the ReggePonzano model as follows.

The state space $V_{C S W}(F)$ of the $I S O(3)$ theory consists of functionals $\psi(\omega)$ on the moduli space of flat $S O(3)$ connections on a 2 -surface $F$. The wave function for a particular manifold $M$ is given by the functional $\psi_{M}(\omega) \equiv\left\langle\omega_{i} \mid \psi_{M}\right\rangle$ defined by the path integral (2.10), with boundary condition that $\omega_{i}$ is the flat connection on the boundary $\partial M=F$. There is a natural inner product on this space given by

$$
\left\langle\psi_{1}(\omega) \mid \psi_{2}(\omega)\right\rangle=\int_{F}[d \omega] \delta\left(F_{i j}\right) \overline{\psi_{1}(\omega)} \psi_{2}(\omega) .
$$

It follows that the inner product of two Chern-Simons wave functions corresponding to two handlebodies $M_{1}$ and $M_{2}$ is given by the partition function for the closed, oriented manifold $M$ formed by gluing together the two handlebodies,

$$
\left\langle\psi_{M_{1}}(\omega) \mid \psi_{M_{2}}(\omega)\right\rangle=Z_{C S W}\left(M_{2} \cup M_{1}\right) .
$$

Ooguri [1992] related the two theories by constucting the trivalent graph $W$, dual to the triangulation $\Delta$ on the 2-dimensional boundary $\partial M$. Each edge $C_{i}$ of this graph is 
labelled by the representation $j_{i}$ on the edge of the triangulation that it crosses. This graph is interpreted as a Wilson line network by assigning, to edge $C_{i}$, the Wilson line

$$
U_{j_{i}}\left[A, C_{i}\right]=\mathrm{P} \exp \left\{\int_{C_{i}} A_{a}{ }^{I} t_{j_{i}}{ }^{J} \mathrm{~d} x^{a}\right\},
$$

where $t_{j_{i}}$ are the $S O(3)$ generators in the $j_{i}$ representation. The product of these Wilson lines, contracted by $3 j$ symbols at trivalent vertices, gives a gauge-invariant function $\psi_{\Delta, c}(\omega)$ of the triangulation $\Delta$, the colouring $c$ and the flat connection $\omega$. This may be interpreted as a change of basis function

$$
\langle\omega \mid \Delta, c\rangle \equiv \psi_{\Delta, c}(\omega)
$$

between a Regge-Ponzano state $\left|\psi_{M}\right\rangle=\sum_{c}|\Delta, c\rangle\left\langle\Delta, c \mid \psi_{M}\right\rangle \in V_{R P}(F)$ and a ChernSimons wave-function $\left\langle\omega \mid \psi_{M}\right\rangle \equiv \psi_{M}(\omega) \in V_{C S W}(F)$, as

$$
\langle\omega \mid \psi\rangle=\sum_{c \in C(\Delta)}\langle\omega \mid \Delta, c\rangle\langle\Delta, c \mid \phi\rangle
$$

where the sum is over all colourings $c$ of the (fixed) triangulation $\Delta$ on $F$.

Ooguri showed that this correspondance is 1-1 and independent of the triangulation chosen, and so the state spaces of the two theories $V_{R P}(F)$ and $V_{C S W}(F)$ are isomorphic. He also claimed that the two inner products given by (2.9) and (2.12) are equivalent, and so the Regge-Ponzano theory and the $I S O(3)$ Chern-Simons theory are equivalent as physical theories, (subject to the proviso that neither theory is mathematically well-defined). This result suggests that the Regge-Ponzano theory may be interpreted as providing a model for quantum gravity in three dimensions, and that we should look for a similar result for the well-defined Turaev-Viro theory. 


\section{Spin networks and the inner product.}

Spin networks were introduced by Penrose [1971] as a discrete model underlying spacetime. A spin network is an $S U(2)$ invariant tensor represented by a trivalent graph, a network with three edges meeting at each vertex. Each edge is labelled by a spin- $j$

representation of the angular momentum covering group $S U(2)$, where $j \in 0, \frac{1}{2}, 1, \ldots$ For the spin network to be non-zero, the triangle inequalities must hold at each vertex,

$$
\left|j_{2}-j_{3}\right| \leq j_{1} \leq j_{2}+j_{3}
$$

and also, the sum of the spins must be integral,

$$
j_{1}+j_{2}+j_{3} \in \mathbf{Z}
$$

where $j_{1}, j_{2}, j_{3}$ are the spins assigned to the three edges meeting at that vertex.

Penrose showed how to evaluate a spin network by using the binor calculus [Penrose 1972]. In this approach, a strand network is associated with each spin network by replacing each edge, labelled by spin $j$, by a linear combination of $2 j$ strands, and summing over all ways of joining these strands at each vertex. So, a vertex is replaced in the strand picture by

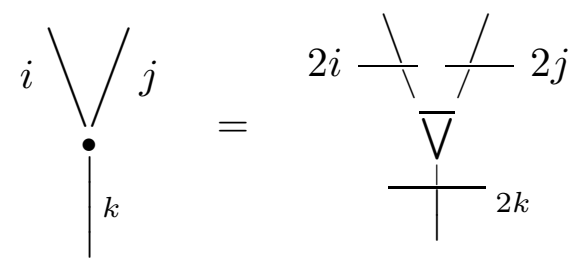

where each bar represents an anti-symmetriser, given by the linear combination of the different ways the $2 j$ strands may cross, with a co-efficient of $(-1)$ for each crossing, e.g.

$$
\begin{aligned}
& r^{2}=\|-X \\
& \frac{1}{1}^{3}=\||-| X-X \mid+X+X-X
\end{aligned}
$$

An oriented abstract spin network (where the orientation may be given by the embedding of the spin network in the plane) is evaluated by decomposing each strand network into a number of closed loops using the two basic binor identities [Penrose 1971, 1972],

$$
\begin{aligned}
& \left.X=-\bigcup_{\bigcap}-\right)( \\
& \bigcirc=-2 .
\end{aligned}
$$


These identities form the basis of a topologically invariant diagrammatic calculus [Kauffman 1990], based on the $\mathrm{SU}(2)$ invariant tensor $\varepsilon_{A B}$ represented by

$$
\bigcap=i \varepsilon_{A B}=i\left(\begin{array}{cc}
0 & 1 \\
-1 & 0
\end{array}\right) \text {. }
$$

The binor calculus provides a way of calculating the norm of an abstract oriented spin network, which only depends on the network as an abstract graph together with its orientation. As we shall see in the next section, Kauffman [1991] showed that the binor identity is a special case of his bracket identity, which can be used to define a spin network based on the quantum group $s l(2)_{q}$.

We can now use spin networks to look at the relation of the Regge-Ponzano model to the loop representation of quantum general relativity [Rovelli and Smolin 1989], in a similar way to the work of Rovelli [1993]. Rovelli related Ooguri's Wilson line network to the loop representation as follows. Each Wilson line $U_{j_{i}}\left[A, C_{i}\right]$ in the $j$ representation is replaced by $2 j$ Wilson lines in the $\frac{1}{2}$ representation, and each trivalent intersection is replaced by the sum over all ways of joining the strands. The strand network corresponding to a coloured triangulation $(\Delta, c)$ is thus an ensemble of multiple loops, $E_{\Delta, c}=\left\{\alpha_{1}, \alpha_{2}, \ldots\right\}$, where each multiple loop $\alpha_{i}$ has the property that $2 j$ single loops cross a link of the triangulation with colour $j$.

Here, we interpret the dual graph to a coloured triangulation in the Regge-Ponzano model as a spin network. This was first suggested by Hasslacher and Perry [1981] and Moussouris [1983] for the case of a spin network on $S^{2}$. This spin network is converted to a strand network by replacing each trivalent vertex by a sum over strands according to (3.3). This strand network is then evaluated using the binor relations (3.5),(3.6). The key difference here from Rovelli's approach is that the presence of the extra negative signs in the anti-symmetrisers (3.4). This rule, together with the binor relations, ensures that the evaluation of the spin network is topologically invariant. We shall see in the next section that this is because the binor relations $(3.5),(3.6)$ are a special case of the Kauffman relations.

Now, the state space of the Regge-Ponzano model $V_{R P}(F)$ is given by the space of linear combinations of colourings of a triangulation. We map a particular coloured triangulation to its dual spin network, by mapping a coloured edge of the triangulation to an edge of the network crossing it with the same colouring, as follows

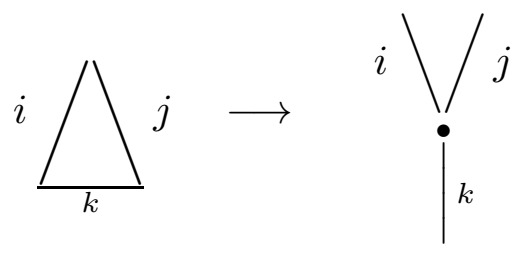


It is clear that this mapping is 1-1. A spin network is evaluated as a linear combination of strand networks which obey the binor identities and so are isotopy invariant. This suggests that we can re-write the state space $V_{R P}(F)$ as the space of linear combinations of isotopy classes of links, quotiented by the binor relations. As we shall see in the next section, this is an example of a skein space.

A basis element in the Regge-Ponzano state space $V_{R P}(F)$ given by a paticular colouring of a triangulation $\left|\Delta, c_{i}\right\rangle$ maps to a spin network state $\left|s_{i}\right\rangle$. A spin network state corresponds to a linear combination of strand network states $\left|\alpha_{i}\right\rangle$ as above,

$$
\left|\Delta, c_{i}\right\rangle \rightarrow\left|s_{i}\right\rangle=\sum_{\alpha_{i} \in s_{i}}(-1)^{n}\left|\alpha_{i}\right\rangle
$$

where $n$ is the number of crossings in that state. We now map a strand network state to an equivalence class of a multiple loop under the binor relations (3.5),(3.6). So, a spin network $\left|s_{i}\right\rangle \in V_{R P}(F)$ maps to a state in the space $V_{\text {loop }}(F)$ of complex linear combinations of characteristic functionals on isotopy classes of loops, quotiented by the binor relations.

We now want to consider the question of how to define an inner product on the state space. Rovelli proposed that mappings of the above type could be used to define the inner product between two loop states using Regge-Ponzano theory. From the above formulation, we propose the following interpretation. As a coloured triangulation maps 1-1 on to a spin network, this suggests that, following (2.6), we should take spin network states with different colourings to be orthonormal,

$$
\left\langle s_{i} \mid s_{j}\right\rangle=\delta_{i j}
$$

An arbitrary loop state $\left|\gamma_{i}\right\rangle \in V_{\text {loop }}(F)$ may be written as a linear combination of spin network states $\left|s_{j}\right\rangle$ as

$$
\left\langle\gamma_{i}\right|=\sum_{j} \lambda_{j}\left\langle s_{j}\right|
$$

For a particular state corresponding to the vector for a 3-manifold, $\left|\gamma_{M}\right\rangle$, the co-efficient is $\lambda_{j}=Z\left(M, s_{j}\right)$, and so

$$
\left\langle\gamma_{M_{1}} \mid \gamma_{M_{2}}\right\rangle=Z_{R P}\left(M_{1} \cup M_{2}\right)
$$

We shall discuss in section 5, the comparison between this approach and that of Rovelli and Smolin [1990]. 


\section{Turaev-Viro theory and skein space.}

I now want to extend the above work to begin to investigate the relation between the topological field theory of Turaev and Viro [1992] and the loop representation of quantum general relativity in 3 dimensions. There are two pieces of evidence which lead us to suspect such a relation. Firstly, Turaev-Viro theory is the generalisation of the ReggePonzano theory in which the representations of the classical group $S U(2)$ are replaced by those of a quantum group $U_{q}(s l(2))$ at $q$ an $r$-th root of unity, so that the partition function is a finite sum. Thus, we may expect that the Turaev-Viro theory would be related to the loop representation. Secondly, we know that the Turaev-Viro invariant is the square of the Chern-Simons-Witten invariant for gauge group $S U(2)$, which is equivalent to the $S O(4)$ Chern-Simons theory that is related to 3-dimensional Euclidean gravity with a positive cosmological constant. This formal relation was implicit in Witten's work and was first written explicitly in [Ooguri and Sasakura 1991] and [Archer and Williams 1991], as follows

$$
\begin{aligned}
Z_{T V, r}(M) & =\left|Z_{C S W, k}(M)\right|^{2} \\
& =\int[\mathrm{d} A, \mathrm{~d} B] \exp \left(i \frac{k}{4 \pi} \int\left(A \mathrm{~d} A+\frac{2}{3} A^{3}\right)-\left(B \mathrm{~d} B+\frac{2}{3} B^{3}\right)\right) \\
& =\int[\mathrm{d} e, \mathrm{~d} \omega] \exp \left(i \int\left(e \wedge R+\frac{\Lambda_{k}}{3} e \wedge e \wedge e\right)\right),
\end{aligned}
$$

where

$$
\begin{aligned}
\omega & =\frac{1}{2}(A+B), \quad e=\frac{k}{8 \pi}(A-B), \\
\Lambda_{k} & =\left(\frac{4 \pi}{k}\right)^{2},
\end{aligned}
$$

for connections $A, B$ taking values in the Lie algebra of $S U(2)$, connection $\omega$ taking values in $S O(3)$, triad $e$ and cosmological constant $\Lambda_{k}$, for level $k$ of the Chern-Simons theory, which is related to the level $r$ of the Turaev-Viro theory by

$$
k=r-2 .
$$

Much work has been undertaken by mathematicians to provide a more mathematically rigorous proof of the Chern-Simons-Witten topological field theory with gauge group $S U(2)$ which does not rely on the use of path integrals. Reshetikhin and Turaev [1991] defined a topological invariant based on representations of the quantum group $U_{q}(s l(2))$ which satisfies the same formal properties and so may be identified with the Chern-Simons-Witten invariant. In a series of papers, Lickorish $[1991,1993]$ showed that this Witten-ReshetikhinTuraev (W-R-T) invariant could be reproduced using skein theory. Independently, both 
Turaev [1992a,b] and Walker [1992] showed that the Turaev-Viro invariant is the square of the W-R-T invariant, and so justified equation (4.1). More insight into the relation between these two theories was provided by the work of Justin Roberts [Roberts 1993,1994] extending the skein-theoretic approach, and here we shall extend this approach.

In a physical model, we are interested in finding an interpretation of the vector space of states and the inner product between states defined by a topological field theory. From the relation (4.1) between the partition functions and the formalism of a topological field theory [Atiyah 1990], it is expected that the state space for the Turaev-Viro theory to be isomorphic to endomorphisms of the state space for the Witten theory,

$$
V_{T V}(F) \cong \text { End } V_{W R T}(F)
$$

An outline proof of this theorem was given in [Turaev 1992b]. We show how this relation is recovered in the skein-theoretic approach.

The skein space $\mathcal{S} M$ of an oriented 3 -manifold $M$ is the vector space of formal linear sums, over $\mathbf{C}$, of isotopy classes of framed links $L$ in $M$, quotiented by the Kauffman relations [Kauffman 1991]

$$
\begin{gathered}
\left.\searrow /=A \underset{\bigcap}{\cup}+A^{-1}\right)( \\
\bigcirc \cup L=\left(-A^{2}-A^{-2}\right) L,
\end{gathered}
$$

where $\bigcirc$ is a closed contractible loop, and the diagrams in (4.5) represent pieces of links inside a 3-ball, outside of which the links are identical. Applying (4.5) to a link reduces that link to a linear combination of loops with no crossings inside any local 3-ball, and each contractible loop may be replaced by a co-efficient by (4.6). Thus, the content of the skein space is that it is the space of linear combinations of closed loops with no local crossings which are both non-contractible and disjoint.

We can see that the Penrose binor relations (3.5),(3.6) are a special of the Kauffman skein relations, given by taking $A=-1$.

It is convenient to use a two-dimensional projection to describe the skein space, but we can see that the above definition is inherently three-dimensional. In particular, the skein space $\mathcal{S} A$ of a solid torus $S^{2} \times S^{1}$ is described by its projection on to a 2-dimensional annulus $A$ and is given by the polynomial algebra over $\alpha_{n}$, where $\alpha_{n}$ is the basis element consisting of $n$ loops around the hole. We can also use this description as a projection to define the skein space $\mathcal{S} F$ of a 2 -surface $F$ as the skein space of the cylinder $F \times[0,1]$ over the surface. 
A description of the state space of a surface in the W-R-T theory using skein theory was given by Roberts [1994] as follows. Consider the surface $F$ as dividing $S^{3}$ into a handlebody $H$ and its dual $H^{\prime}$,

$$
S^{3}=H \cup(F \times I) \cup H^{\prime},
$$

and define the bilinear form

$$
\langle,\rangle: \mathcal{S} H \times \mathcal{S} H^{\prime} \rightarrow \mathcal{S} S^{3}=\mathbf{C},
$$

then, on the quotient $\mathcal{H}$ of the skein space of the handlebody defined by

$$
\begin{aligned}
U & =\operatorname{Ker}\left(\mathcal{S} H \rightarrow\left(\mathcal{S} H^{\prime}\right)^{*}\right) \\
\mathcal{H} & =\mathcal{S} H / U
\end{aligned}
$$

for $q$ a $2 r$-th root of unity, the form descends to a non-degenerate pairing

$$
\mathcal{H} \times \mathcal{H}^{\prime} \rightarrow \mathbf{C} .
$$

This implies [Blanchet et al 1993] that the space $\mathcal{H}$ is finite dimensional and may be identified as the state space of the W-R-T theory

$$
V_{W R T}(F) \cong \mathcal{H}, \quad(F=\partial H) .
$$

We now consider the $q$-deformed analogues of spin networks [Kauffman 1991,1992]. As in the undeformed 'classical' case, a $q$-spin network is a labelled trivalent graph, but now the labellings refer to integer representations of the quantum group $s l(2)_{q}$. Again, a trivalent vertex or triad corresponds to a linear combination of connected strands, but the anti-symmetriser is now replaced by a $q$-symmetriser or idempotent $f^{(j)}$. This represents a linear combination of braided strands, i.e. one strand locally overcrosses another but the strands do not intersect. So, a triad $T(i, j, k)$ is an element of the skein space of the form

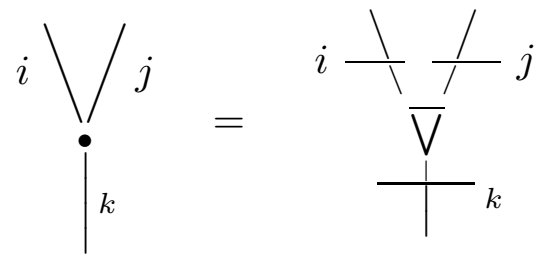

where a $q$-symmetriser is given by the linear combination of the different ways of braiding the $2 j$ strands with a co-efficient of $\left(+A^{-3}\right)$ for each crossing, e.g.

$$
\begin{aligned}
& \left.\underline{I}^{2}=\|+A^{-3}\right\rangle^{\prime} \\
& \left.\underline{I}^{3}=\|\left|+A^{-3}\right| \bigwedge^{\prime}+A^{-3}\right\rangle^{\prime}\left|+A^{-6} \lambda\right|^{\prime}+A^{-6} \lambda^{/ /}+A^{-9} \bigvee^{\prime}
\end{aligned}
$$


In the limit $A \rightarrow-1$, which corresponds to $q=A^{2} \rightarrow 1$, these reduce to the antisymmetrisers (3.4), and so a $q$-spin network reduces to a classical spin network as $q \rightarrow 1$.

A $q$-spin network is evaluated by applying the Kauffman relations (4.5), (4.6) to each crossing and contractible loop, and so is naturally an element of the skein space $\mathcal{S} M$. A $\operatorname{triad} T(i, j, k)$ is defined for

$$
\begin{aligned}
i, j, k & \in\{0,1, \ldots, r-1\}, \\
i \leq j+k, j & \leq i+k, k \leq i+j, \quad \frac{1}{2}(i+j+k) \in \mathbf{Z},
\end{aligned}
$$

and is said to be admissble if

$$
\begin{aligned}
i, j, k & \leq r-2, \\
\frac{1}{2}(i+j+k) & \leq(r-2),
\end{aligned}
$$

and inadmissible if these relations are not satisfied. Note that networks are labelled by integers, whereas triangles in the Turaev-Viro picture were labelled by half-integers.

A $q$-spin network is admissible if and only if all its triads are admissible. As we shall see, we want to impose the condition that we only consider admissible networks. So, we define $N$ as the subspace of the skein space $\mathcal{S} M$ of any 3-manifold generated by inserting an inadmissible triad anywhere into the manifold $M$, i.e. the subspace of isotopy classes of $q$-spin networks in $M$ for which any triad is inadmissible, and quotient out by this subpsace. Roberts [1994] showed that this quotient space of the skein space of a handlebody,

$$
\mathcal{H}=\mathcal{S} H / N,
$$

which we call the reduced skein space, is equivalent to the quotient space (4.10). So, the state space for the Witten theory $V_{W R T}(F) \cong \mathcal{H}$ has now been written as the quotient space of the skein space of the handlebody by the subspace generated by inadmissible triads. We can regard this as the space of admissible $q$-spin networks on the handlebody.

Furthermore, Roberts showed that the skein space $\mathcal{S} F$ of the 2-surface $F$ maps surjectively onto the endomorphisms of $\mathcal{H}$, and so the quotient $\mathcal{F}$ of the skein space by the kernel of this map

$$
\begin{aligned}
Q & =\operatorname{Ker}(\mathcal{S} F \rightarrow(\operatorname{End} \mathcal{H})) \\
\mathcal{F} & =\mathcal{S} F / Q
\end{aligned}
$$

is isomorphic to the algebra of endomorphisms of $\mathcal{H}$,

$$
\mathcal{F} \cong \text { End } \mathcal{H} \text {. }
$$


Comparing this relation with the expected relation (4.4) between the state spaces of the two theories, I conjecture that space $\mathcal{F}$ is isomorphic to the state space of the TuraevViro theory $V_{T V}(F)$, and further that, in analogy with Roberts' result for the Witten state space, this space $\mathcal{F}$ is equivalent to the reduced skein space defined by

$$
\mathcal{F}=\mathcal{S} F / N,
$$

where $N$ is the subspace of $\mathcal{S} F$ generated by inserting any inadmissible triad into $F$.

This relation may be expected from general principles. Recall that the Turaev-Viro theory [Turaev and Viro 1992] is specified by the partition function (2.1), which is given by a state sum over admissible colourings of a triangulated 3-manifold. The labels $(i, j, k)$ assigned to the three edges around any triangle must satisfy

$$
\begin{aligned}
& (i, j, k) \in\left\{0, \frac{1}{2}, 1, \ldots, \frac{(r-2)}{2}\right\}, \\
& i \leq j+k, j \leq i+k, k \leq i+j, \quad i+j+k \in \mathbf{Z},
\end{aligned}
$$

and the admissibility condition

$$
i+j+k \leq(r-2),
$$

where the theory is defined at $q$ an $r$-th root of unity.

The state space $V_{T V}(F)$ of the Turaev-Viro theory is defined as the space of linear combinations of admissible colourings $C(F)$ on the triangulated surface $F$, quotiented by the kernel of the isomorphism $\Phi_{(F \times I)}$ corresponding to the cylinder $F \times[0,1]$ over the surface,

$$
V_{T V}(F)=C(F) / \operatorname{Ker} \Phi_{(F \times I)} .
$$

An admissible colouring of a triangulation of the surface, $|\Delta, c\rangle \in V_{T V}(F)$, may be mapped to a $q$-spin network, as follows. Take the trivalent graph on the surface dual to the triangulation, as in (3.8), and label an edge of the graph by twice the half-integer label on the edge of the triangulation which it crosses. This integer-labelled trivalent graph may now be interpreted as a $q$-spin network. Furthermore, a colouring of a triangulation which is admissible according to (4.21), (4.22) maps to a network in which the colouring of each triad is admissible by (4.16). Thus, the map from the Turaev-Viro state space $V_{T V}(F)$ to the reduced skein space $\mathcal{F}$ given by

$$
\phi: V_{T V}(F) \rightarrow \mathcal{F}
$$

is surjective and we expect it to be an isomorphism. 
To gain more physical insight into the nature of the reduced skein space $\mathcal{F}$, we may attempt to find a basis for this space. A basis is not given simply by the set of all admissible colourings of a $q$-spin network dual to a simple triangulation of the surface, as there is a subtlety due to the fact that this space is the skein space quotiented by the space generated by inadmissible triads.

Let us consider the simple example in which $F$ is the surface of the torus $S^{1} \times S^{1}$ and $r=4$ so that the admissible colourings of an edge are $j \in\{0,1,2\}$. The Witten state space for this surface is the reduced skein space $\mathcal{H}$ of the solid torus, which has a basis $\alpha_{j}$ given by $j \in\{0,1,2\}$ loops around the hole, (since the inadmissibility of triads of higher order imply that the skein element for any higher number of loops must be zero),

$$
\mathcal{H}=\left\{\sum_{j} \lambda_{j} \alpha_{j}: \lambda_{j} \in \mathbf{C}, j \in\{0,1,2\}\right\}
$$

and so is 3-dimensional. By the relation (4.4), the Turaev-Viro state space $V_{T V}(F)$ should be 9-dimensional. The simplest (degenerate) triangulation of the surface consists of two triangles with their edges identified and so the dual $q$-spin network has two vertices and three edges, labelled by $j_{1}, j_{2}, j_{3}$, such that $\left(j_{1}, j_{2}, j_{3}\right)$ form an admissible triad. In this case, the admissible colourings of a triad are $\{(0,0,0),(0,1,1),(0,2,2),(1,1,2)\}$ and so, allowing for permutations, there are 10 admissible colourings of this network. However, these colourings are not independent in the reduced skein space $\mathcal{F}$ because of relations generated by inadmissible colourings. In particular, we find that there is exactly one relation which may be generated by the inadmissible colouring $(2,2,2)$ of this network, and so the space $\mathcal{F}$ is indeed 9-dimensional. Similarly, we find that, for the case $r=5$, there are four relations amongst the 20 admissible colourings of this network and so $\mathcal{F}$ is 16-dimensional. These examples lend support to our conjecture that $\mathcal{F}$ may be identified as the Turaev-Viro state space $V_{T V}(F)$.

Comparing with the definition (2.3) for the Regge-Ponzano theory, this suggests that we could define an inner product on the reduced skein space by taking different colourings of a $q$-spin network to be orthonormal. This picture of the state space should also enable us to relate a $q$-spin network on the boundary to the the Kauffman-Lins [1990] formulation of the Turaev-Viro theory in the interior of the 3-manifold. 


\section{Discussion.}

In this paper, we have explored several aspects of the relation between spin networks, simplicial state-sum models and the loop representation for quantum general relativity. We conclude by summarising what we have learned and discussing the possible implications.

We have given descriptions of the state space of a surface for the Ponzano-Regge theory (2.3) as the space of spin networks on that surface, and for the Turaev-Viro theory as the space of admissible $q$-spin networks on the surface (4.24). The latter space is called the reduced skein space of the surface. The advantage of working with skein space is that isotopy invariance is automatically encoded in the formalism. Indeed, the Kauffman skein relations underly the Jones polynomial isotopy invariant (see [Kauffman 1991]). The other advantage is that the reduced skein space is isomorphic to endomorphisms the state space for the Witten theory based on $S U(2)$, as expected from the fact that the Turaev-Viro theory is the square of that theory.

We also considered the definition of the inner product for the Regge-Ponzano theory. In any topological field theory, there corresponds a vector, in the state space for a surface, to a manifold with that surface as its boundary. The inner product defined on the state space must be such that the inner product of the two vectors corresponding to two manifolds which meet in a common surface is given by the invariant of the manifold given by their union. This guarantees that the invariant of a closed manifold does not depend on how the manifold is cut into two pieces (see [Atiyah 1990]), and so this inner product carries a great deal of information about the elements of the theory. We saw that, for the Regge-Ponzano theory, we could recover this topological inner product from the inner product defined by taking different colourings of a particular spin network on the surface to be orthonormal.

Building on the work of Ooguri [1992] and Rovelli [1993], we considered the relation between the Regge-Ponzano theory and the loop representation for quantum general relativity. As emphasized by Ashtekar [1991], the problem of finding an inner product on the space of states is one of the key problems in non-perturbative canonical gravity. Rovelli and Smolin [1990] defined the state space for quantum general relativity as a quotient of the space of functionals on multiple loops. Such loop states were related by the $S U(2)$ spinor relations, from which the binor relations differ in the sign of the terms. They showed that states given by functionals on link isotopy classes of simple, non-intersecting loops satisfied the constraint equations, and so could be identified as physical states. They defined an inner product by taking characteristic functionals on isotopy classes of simple loops to be orthonormal, (see Smolin [1992]).

Here, we took the loop state space to be the space of complex linear combinations 
of isotopy classes of loops, and wrote an arbitrary loop state as a linear combination of spin network states. We defined the inner product by taking different colourings of a spin network to be orthonormal. The advantages of this approach are that isotopy invariance is automatically encoded by the binor relations which underly spin networks, and that the inner product is consistent with the topological inner product, as explained above. However, it is not clear whether suitable self-adjoint operators may be defined under this inner product, and so this interpretation must be regarded as provisional.

The above considerations, together with the work of Ooguri [1992], suggest that the Regge-Ponzano theory may be regarded as a discrete model for quantum gravity in three dimensions. Unfortunately, this theory is not mathematically well-defined as the state sum (2.2) is infinite and it must be regularised. Ooguri followed the regularisation of Ponzano and Regge, but it is not clear how rigorous this is. We have ignored these problems here and treated the Regge-Ponzano theory as a formal topological field theory. It is actually a generalisation of such a theory in the sense that the state space $V_{R P}(F)$ is infinitedimensional. Rovelli [1993] suggested that quantum general relativity in four dimensions may be a generalised topological field theory of this kind.

However, at least in three dimensions, these problems with regularisation may be completely overcome by considering instead the well-defined Turaev-Viro theory. The state sum (2.1) is finite as there are only a finite set of edge labels (4.21) and so of possible colourings, for the theory defined at $q$ an $r$-th root of unity. As $q \rightarrow 1$ and $r \rightarrow \infty$, the Turaev-Viro theory reduces to the Ponzano-Regge theory and so it may be regarded as a naturally regularised version of that theory. The Turaev-Viro state space $V_{T V}(F)$ is finitedimensional, and we have conjectured that it is isomorphic to the reduced skein space of the surface $F$. As $r \rightarrow \infty$, the reduced skein space goes to the space of linear combinations

of isotopy classes of links quotiented by the binor relations, which we identified as the Regge-Ponzano state space.

This suggests that we may interpret the Turaev-Viro theory as a finite discrete model for quantum gravity. As with the Ponzano-Regge theory, we may define the inner product by taking different colourings of a $q$-spin network to be orthonormal. We would then need to check that this definition was equivalent to the topological inner product. If so, it would be interesting to consider whether the Turaev-Viro theory could be related to the loop representation.

\section{Acknowledgements.}

I would like to thank John Barrett, Chris Fewster and Justin Roberts for helpful comments and discussions. 


\section{References.}

F.J. Archer and R.M. Williams 1991, "The Turaev-Viro state-sum model and 3-dimensional quantum gravity," Phys. Lett. B273 (1991), 438-450.

A. Ashtekar 1991, Non-perturbative canonical gravity, lecture notes prepared with R.S. Tate, (World Scientific, Singapore, 1991).

M. Atiyah 1990, The geometry and physics of knots, (Cambridge University Press, 1990).

C. Blanchet, N. Habegger, G. Masbaum and P. Vogel 1993, "Topological quantum field theories derived from the Kauffman bracket," preprint, University of Nantes, 93-02/1.

B. Hasslacher and M.J. Perry 1981, "Spin networks are simplicial quantum gravity," Phys. Lett. 103B (1981), 21-24.

L.H. Kauffman 1990, "Spin networks and knot polynomials," Int. J. Mod. Phys. A5 (1990), 93-115.

L.H. Kauffman 1991, Knots and physics, (World Scientific, Singapore, 1991).

L.H. Kauffman 1992, "Map colouring, $q$-deformed spin networks, and Turaev-Viro invariants for 3-manifolds," Int. J. Mod. Phys. B6 (1992), 1765-1794.

L.H. Kauffman and S. Lins 1990, "A 3-manifold invariant by state summation," preprint, University of Illinois at Chicago, (1990).

W.B.R. Lickorish 1991, "Three-manifolds and the Temperley-Lieb algebra," Math. Ann. 290 (1991), 657-670.

W.B.R. Lickorish 1993, "The skein method for three-manifold invariants," J. Knot Theory and its Ramifications 2 (1993), 171-194.

J.P. Moussouris 1983, "Quantum models of space-time based on recoupling theory", D.Phil. Thesis, (Oxford University, 1983).

H. Ooguri 1992, "Partition functions and topology-changing amplitudes in the 3D lattice gravity of Ponzano and Regge," Nucl. Phys. B382 (1992), 276-304.

H. Ooguri and N. Sasakura 1991, "Discrete and continuum approaches to three-dimensional quantum gravity," Mod. Phys. Lett. A6 (1991), 3591-3600.

R. Penrose 1971, "Angular momentum: An approach to combinatorial space-time," in Quantum theory and beyond, ed. T.Bastin, (C.U.P., Cambridge, 1971).

R. Penrose 1972, "Applications of negative dimensional tensors," in Combinatorial theory and applications, ed. D. Welsh, (Wiley, 1972).

G. Ponzano and T. Regge 1968, "Semiclassical limit of Racah coefficients," in Spectroscopic and group theoretical methods in physics, ed. F. Bloch, (North Holland, 1968). 
N.Y. Reshetikhin and V.G. Turaev 1991, "Invariants of 3-manifolds via link polynomials and quantum groups," Invent. Math. 103 (1991), 547-597.

J.D. Roberts 1993, "Skein theory and Turaev-Viro invariants," preprint, DPMMS, Cambridge.

J.D. Roberts 1994, "Skeins and mapping class groups," Math. Proc. Camb. Phil. Soc. 115 (1994), 53-77.

C. Rovelli 1993, "Basis of the Ponzano-Regge-Turaev-Viro-Ooguri quantum gravity model is the loop representation basis," Phys. Rev. D 48 (1993), 2702-2707.

C. Rovelli and L. Smolin 1990, "Loop representation for quantum general relativity," Nucl. Phys. B331 (1990), 80-152.

L. Smolin 1991, "Recent developments in non-perturbative quantum gravity," in Proceedings of the 1991 GIFT International Seminar on Quantum Gravity and Cosmology, (World Scientific, Singapore, in press).

V.G. Turaev 1992a, "Quantum invariants of 3-manifolds and a glimpse of shadow topology," Lect. Notes in Math. 1510, 363-366.

V.G. Turaev 1992b, "Quantum invariants of 3-manifolds," preprint, Institut de Recherche Mathématique Avancée, Strasbourg.

V.G. Turaev and O.Y. Viro 1990, " State sum invariants of 3-manifolds and quantum $6 j$ symbols," Topology 31 (1992), 865-902.

K. Walker 1992, "On Witten's 3-manifold invariants," preprint.

E. Witten 1989a, "Quantum field theory and the Jones polynomial," Commun. Math. Phys. 121 (1989), 351-399.

E. Witten 1989b, "2+1 dimensional gravity as a exactly soluble theory," Nucl. Phys. B311 (1989), 46-78.

E. Witten 1989c, "Topology-changing amplitudes in 2+1 dimensional gravity," Nucl. Phys. B323 (1989), 113-140. 